Canad. Math. Bull. Vol. 21 (4), 1978

\title{
LIMIT DISTRIBUTIONS FOR THE EXTREME ORDER STATISTICS
}

\author{
BY \\ D. MEJZLER
}

1. Introduction. Let $X_{1}, \ldots, X_{n}$ be independent random variables with the same distribution function $(d f) F(x)$ and let $X_{1 n} \leq X_{2 n} \leq \cdots \leq X_{n n}$ be the corresponding order statistics. The $d f$ of $X_{k n}$ will be denoted always by $F_{k n}(x)$.

Many authors have investigated the asymptotic behaviour of the maximal term $X_{n n}$ as $n \rightarrow \infty$. Gnedenko [3] proved the following

THEOREM 1.1. A proper $d f$ can be a limit of a sequence of the form $\left\{F_{n n}\left(a_{n} x+\right.\right.$ $\left.\left.b_{n}\right)\right\}$, where $\left\{a_{n}, b_{n}\right\}$ are numerical sequences, if and only if it belongs to a type, whose representative is given by a df of one of the following three forms:

$$
\begin{array}{cc}
\Lambda(x)=\exp \left(-e^{-x}\right) & \\
\Phi(x ; \gamma)=\exp \left(-x^{-\gamma}\right), & (x>0), \\
\Psi(x ; \gamma)=\exp \left(-|x|^{\gamma}\right), & (x<0),
\end{array}
$$

where $\gamma>0$ is a constant.

This class of $d f^{\prime} s$ will be denoted here by $A$.

Smirnov [6] considered the term $X_{k n}$, where $k=k(n)$ is a function of $n$ and he investigated the possible proper limits of a sequence of the form

$$
\left\{F_{k n}\left(a_{n} x+b_{n}\right)\right\}
$$

under the assumption that $k / n \rightarrow \lambda$ as $n \rightarrow \infty$. A sequence of terms $X_{k n}$ was called a sequence of central terms if $0<\lambda<1$, and extreme terms if $\lambda=0$ or $\lambda=1$, respectively. By the additional condition

$$
\sqrt{ } n(k / n-\lambda) \rightarrow t, \quad(n \rightarrow \infty), \quad|t|<\infty,
$$

Smirnov found the class of all limits for the central terms. But the investigation of sequences of extreme terms was restricted only to the cases when $k=$ Const $(\lambda=0)$ or, what is essentially the same, when $p=n-k(n)=$ Const. $(\lambda=1)$.

The behaviour of the extreme terms, when simultaneously

$$
k(n) \rightarrow \infty \text { and } p(n)=n-k(n) \rightarrow \infty
$$

Received by the editors August 3, 1977 and, in revised form, November 14, 1977. 
was studied from various aspects in $[1,2,7,8]$. We cite here only a result of Chibisov [2] concerning the left extreme terms:

TheOREM 1.2. Let $k$ satisfy condition (1.3) and $k / n \rightarrow 0$. Let in addition

$$
\sqrt{ } k(n+r)-\sqrt{ } k(n) \rightarrow \alpha \nu \ell / 2, \quad(n \rightarrow \infty),
$$

for every sequence of integers $r=r(n)$ such that

$$
r(n) / n^{1-\alpha / 2} \rightarrow \nu,
$$

where $0<\alpha<1, \nu>0$ and $\ell>0$ are constants. Then a proper $d f H(x)$ is a limit of a sequence of the form (1.2) only if

$$
H(x)=N(-\ln |\ln A(x)|),
$$

where $N(x)$ is the standard normal $d f$ and $A(x)$ any $d f$ from the class $A$.

As Chibisov himself noted, the assumptions of his theorem imply that $k(n) / n^{\alpha} \rightarrow \ell^{2}$.

We consider the right extreme terms and we prove the following

THEOREM 1.3. Let the integer valued function $p(n)$ satisfy condition (1.3) and

$$
\begin{gathered}
p(n) / n \rightarrow 0 . \\
\sqrt{ } p(n+1)-\sqrt{ } p(n) \rightarrow 0 .
\end{gathered}
$$

Let $\left\{n_{s}\right\}$ be the increasing sequence of all integers such that $n_{0}=0$, $p\left(n_{s}\right) \neq p\left(n_{s+1}\right)$; let $g(n)$ be the continuous polygonal function, generated by $p(n)$, i.e.

$$
\begin{aligned}
& g(n)=p\left(n_{s}\right)+\left(n-n_{s}\right) \Delta_{s}, \quad n_{s} \leq n \leq n_{s+1} ; \quad \Delta_{s}=\left(p\left(n_{s+1}\right)-p\left(n_{s}\right)\right) /\left(n_{s+1}-n_{s}\right), \\
& s=0,1, \ldots
\end{aligned}
$$

If

$$
[(n+1) g(n)-n g(n+1)] / \sqrt{ } g(n) \rightarrow \infty,
$$

then a proper $d f H(x)$ can be a limit of a sequence of the form (1.2) only if $H(x)$ is given by (1.4).

Our theorem includes functions $p(n)$ of various order of growth and, in particular, such functions as, e.g., $[\log n],[n / \log n]$ or $\left[n^{\alpha}\right]$, where $0<\alpha<1$.

2. Preliminary remarks. By condition (1.6) we have

$$
\sqrt{ } g(n)-\sqrt{ } p(n) \rightarrow 0
$$


and, therefore, by (1.3) and (1.5),

$$
\begin{aligned}
g(n) & \rightarrow \infty \\
g(n) / n & \rightarrow 0 \\
\sqrt{ } g(n+1)-\sqrt{ } g(n) & \rightarrow 0 .
\end{aligned}
$$

If condition (1.8) holds, then for every $d>0$ we have, from some $n$ on,

$$
\frac{g(n+1)}{g(n)}<1+\frac{1}{n}\left(1-\frac{d}{\sqrt{ } g(n)}\right) \text {. }
$$

Let us denote

$$
\bar{u}_{n}(x)=n \frac{F(x)-1}{\sqrt{ } p(n)}+\sqrt{ } p(n) .
$$

It follows from a remarkable proposition of Smirnov ([6], part 1, §4, Lemma 2) that under the conditions (1.3) and (1.5) the relation

$$
F_{k n}\left(a_{n} x+b_{n}\right) \rightarrow H(x)
$$

holds if and only if

$$
\bar{u}_{n}\left(a_{n} x+b_{n}\right) \rightarrow u(x)
$$

and the non-decreasing function $u(x)$ is uniquely determined from $H(x)$ by the equation

$$
H(x)=N(u(x)) .
$$

(Here and in the sequel convergence of sequences of monotone functions means weak convergence i.e. convergence at each point of continuity of the limit function)

It is easy to show that if $g(n)$ is any function, which satisfies condition (2.1) and

$$
u_{n}(x)=n \frac{F(x)-1}{\sqrt{ } g(n)}+\sqrt{ } g(n),
$$

then the sequences $\left\{\bar{u}_{n}\left(a_{n} x+b_{n}\right)\right\}$ and $\left\{u_{n}\left(a_{n} x+b_{n}\right)\right\}$ both diverge or simultaneously converge to the same finite or infinite limit $u(x)$, whatever is the pair $\left\{a_{n}, b_{n}\right\}$. Thus relation (2.6) can be replaced by

$$
u_{n}\left(a_{n} x+b_{n}\right) \rightarrow u(x)
$$

where $u_{n}(x)$ is given by (2.8) and $g(n)$ is the polygonal function (1.8).

By equation (2.7), $u(x)$ must be a non-decreasing function, which assumes finite or infinite values in $(-\infty, \infty)$ and satisfies the conditions

$$
u(-\infty)=-\infty, \quad u(+\infty)=+\infty .
$$

Such function will be called an $s$-function (sf). 
For every $s f u(x)$ we denote

$$
u=\inf \{x: u(x)>-\infty\}, \quad \bar{u}=\sup \{x: u(x)<+\infty\} .
$$

An $s f$ will be called proper if $u<\bar{u}$. It follows from (2.7) that $H(x)$ is a proper $d f$ if and only if $u(x)$ is a proper $s f$.

Thus our problem is to show, that each proper $s f u(x)$, which can be a limit of the sequence (2.9) must be of the form

$$
u(x)=-\ln |\ln A(x)|
$$

where $A(x)$ is a $d f$ from the class $A$, given by (1.1).

The notion of type of $s f$ 's can be introduced in the same way, as it was done in the class of $d f$ 's. It is easy to prove the following propositions, which are analogous to the theorems of Khintchine and Gnedenko [4, 110 , Theorem 1 and 2]:

THEOREM 2.1. Let $\left\{u_{n}(x)\right\}$ be a sequence of non-decreasing functions (not necessarily sf's) defined in $(-\infty, \infty)$. If

$$
u_{n}(x) \rightarrow u(x), u_{n}\left(a_{n} x+b_{n}\right) \rightarrow u^{*}(x),(n \rightarrow \infty),
$$

where $u(x)$ and $u^{*}(x)$ are proper sfs, then $u(x)$ and $u^{*}(x)$ belong to the same type.

THEOREM 2.2. For a sequence of non-decreasing functions, defined in $(-\infty, \infty)$, the relations

$$
u_{n}\left(a_{n} x+b_{n}\right) \rightarrow u(x) \quad \text { and } \quad u_{n}\left(\alpha_{n} x+\beta_{n}\right) \rightarrow u(x)
$$

as $n \rightarrow \infty$, where $a_{n}, b_{n}, \alpha_{n}, \beta_{n}$ are real constants and $u(x)$ is a proper $s f$, are satisfied simultaneously if and only if

$$
a_{n} / \alpha_{n} \rightarrow 1, \quad\left(b_{n}-\beta_{n}\right) / a_{n} \rightarrow 0 .
$$

By (2.8), (2.2)-(2.4), $u_{n+1}(x)=A_{n} u_{n}(x)+B_{n}$, where $A_{n} \rightarrow 1, \quad B_{n} \rightarrow 0$. Therefore, by (2.9) we have also

$$
u_{n}\left(a_{n+1} x+b_{n+1}\right) \rightarrow u(x)
$$

If $u(x)$ is a proper $s f$, then by Theorem 2.2 we get

$$
a_{n+1} / a_{n} \rightarrow 1, \quad\left(b_{n+1}-b_{n}\right) / a_{n} \rightarrow 0 .
$$

\section{Auxiliary propositions}

Lemma 3.1. (cf. [5], Lemma 2.3). Let relation (2.9) hold, where $u(x)$ is a proper sf and $g(n)$ satisfies conditions (2.2)-(2.3). Then

(1) The partial limits of the sequence $\left\{b_{n} / a_{n}\right\}$ must be outside of the open interval $(-\bar{u},-u)$, where $u$ and $\bar{u}$ were defined by (2.10). 
(2) If, in addition, $u<0$, then for each subsequence $\left\{n^{\prime}\right\}$ such that $b_{n^{\prime}} \rightarrow b$, $(|b|<\infty)$, we have $a_{n^{\prime}} \rightarrow 0$.

Proof. (1) It follows from the definition (2.8) of $u_{n}(x)$ that

$$
\lim u_{n}(0)=\left\{\begin{array}{lll}
+\infty, & \text { if } \quad F(0)=1 \\
-\infty, & \text { if } \quad F(0)<1
\end{array}\right.
$$

Let us assume that for some subsequence $\left\{n^{\prime}\right\}$

$$
b_{n^{\prime}} / a_{n^{\prime}} \rightarrow \beta,
$$

where $u<-\beta<\bar{u}$. Then by Theorem 2.2

$$
u_{n^{\prime}}\left(a_{n^{\prime}} x\right) \rightarrow u(x-\beta) .
$$

Therefore, for every $\varepsilon>0$ and $\eta>0$ such that $(-\beta-\varepsilon)$ and $(-\beta+\eta)$ are continuity points of $u(x)$ we get

$$
u_{n^{\prime}}\left(-\varepsilon a_{n^{\prime}}\right) \rightarrow u(-\beta-\varepsilon), \quad u_{n^{\prime}}\left(\eta a_{n^{\prime}}\right) \rightarrow u(-\beta+\eta),
$$

which by (3.1) is impossible, since for every $n^{\prime}$

$$
u_{n^{\prime}}\left(-\varepsilon a_{n^{\prime}}\right) \leq u_{n^{\prime}}(0) \leq u_{n^{\prime}}\left(\eta a_{n^{\prime}}\right) .
$$

(2) Assuming $a_{n^{\prime}} \rightarrow a$, where $0<a<\infty$, we get

$$
u_{n^{\prime}}(a x+b) \rightarrow u(x) \text {. }
$$

Hence

$$
u(x)=-\infty \quad \text { if } \quad F(a x+b)<1, \quad u(x)=+\infty \quad \text { if } \quad F(a x+b)=1,
$$

which is impossible, since $u(x)$ is a proper $s f$.

Assuming $a_{n^{\prime}} \rightarrow \infty$ and using Theorem 2.2 we get

$$
u_{n^{\prime}}\left(a_{n^{\prime}} x\right) \rightarrow u(x) \text {. }
$$

Therefore, for every $x, u<x<0$, we get $u(x)=-\infty$, which contradicts the definition of $u$.

LEMMA 3.2. Let $g(n)$ satisfy conditions (2.2)-(2.5) and let (2.5) hold for every $n \geq N_{0}(d)$, where $d>0$ is some constant. If for some $\bar{x}$ and some $N \geq N_{0}(d)$

$$
1-F(\bar{x}) \geq \frac{g(N)}{N}\left(1-\frac{d}{\sqrt{ } g(N)}\right)
$$

then for every $x \leq \bar{x}$, from some $n$ on $(n>N)$

$$
u_{n}(x) \geq u_{n+1}(x) \text {. }
$$

Proof. It is easy to verify, that if for some $x$ and $n$

$$
((1-F(x))[n+1) / \sqrt{ } g(n+1)-n / \sqrt{ } g(n)] \geq \sqrt{ } g(n+1)-\sqrt{ } g(n),
$$


then also inequality (3.3) holds. By (2.5), the last inequality may be rewritten as

$$
1-F(x) \geq \frac{g(n)}{n} E_{n}
$$

where

$$
E_{n}=\sqrt{g(n+1) / g(n)} A_{n} /\left(1-A_{n}\right), \quad A_{n}=n(\sqrt{g(n+1) / g(n)}-1) .
$$

But again by (2.5) we have from some $n$ on

$$
A_{n} \leq \bar{A}_{n}=\frac{\sqrt{ } g(n)}{\sqrt{ } g(n+1)+\sqrt{ } g(n)}\left(1-\frac{d}{\sqrt{ } g(n)}\right),
$$

and therefore

$$
E_{n} \leq \bar{E}_{n}=\sqrt{g(n+1) / g(n)} \bar{A}_{n} /\left(1-\bar{A}_{n}\right) .
$$

It is straightforward to check that

$$
\bar{E}_{n}<1-\frac{d}{\sqrt{ } g(n)} .
$$

Obviously, if (3.2) holds for some $\bar{x}$, then it holds also for every $x \leq \bar{x}$, since $F(x)$ does not decrease. Now, let us denote

$$
f(n)=\frac{g(n)}{n}\left(1-\frac{d}{\sqrt{ } g(n)}\right), \quad(n>N) .
$$

Our Lemma will be proved if we show that $f(n)$ does not increase. Indeed, by (2.5) the ratio $g(n) / n$ does not increase and we get immediately that $f(n) \geq$ $f(n+1)$ if $g(n) \geq g(n+1)$. On the other hand, if $g(n)<g(n+1)$, then

$$
\frac{\sqrt{ } g(n)}{n(n+1)}>\frac{\sqrt{ } g(n)}{n}-\frac{\sqrt{ } g(n+1)}{n+1}
$$

and we again have

$$
\frac{g(n)}{n}-\frac{d \sqrt{ } g(n)}{n}=f(n)>f(n+1)=\frac{g(n+1)}{n+1}-\frac{d \sqrt{ } g(n+1)}{n+1},
$$

since by $(2.5)$

$$
\frac{g(n)}{n}-\frac{g(n+1)}{n+1}>\frac{d \sqrt{ } g(n)}{n(n+1)}
$$

Lemma 3.3. (Cf. [5], Lemma 2.1). Under the conditions of Theorem 1.3 if in addition

$$
u<0<\bar{u} \text { and } u(0-)=u(0+)
$$


then there exists a non-decreasing sequence $\left\{\beta_{n}\right\}$ such that also

$$
u_{n}\left(a_{n} x+\beta_{n}\right) \rightarrow u(x) \text {. }
$$

Proof. First we will prove that for every positive integer valued function $m=m(n)$

$$
\lim \inf \left(b_{n+m}-b_{n}\right) / a_{n} \geq 0 .
$$

Let $0<\bar{x}<\bar{u}$ be any continuity point of $u(x)$ and let $d$ be any positive number such that $d>u(\bar{x})$. We have

$$
u_{n}\left(a_{n} \bar{x}+b_{n}\right)=\frac{n}{\sqrt{ } g(n)}\left[F\left(a_{n} \bar{x}+b_{n}\right)-1+\frac{g(n)}{n}\left(1-\frac{d}{\sqrt{ } g(n)}\right)\right]+d \rightarrow u(\bar{x}) .
$$

Therefore, from some $n$ on,

$$
1-F\left(a_{n} \bar{x}+b_{n}\right)>\frac{g(n)}{n}\left(1-\frac{d}{\sqrt{ } g(n)}\right),
$$

since $u(\bar{x})-d<0$. Thus by Lemma 3.2, from some $n$ on,

$$
u_{n}\left(a_{n} x+b_{n}\right) \geq u_{n+m}\left(a_{n} x+b_{n}\right)
$$

for any $x \leq \bar{x}$ and natural $m$.

The assumptions of our Lemma hold for any subsequence $\left\{n^{\prime}\right\}$. Therefore, in order to simplify the notations, it is enough to prove the impossibility of

$$
\left(b_{n+m}-b_{n}\right) / a_{n} \rightarrow-\beta, \quad a_{n} / a_{n+m} \rightarrow \alpha,
$$

where $0<\beta \leq \infty, 0 \leq \alpha \leq \infty$.

Assuming $\beta=\infty$, for every $M>0$ and $\varepsilon>0$, from some $n$ on,

$$
-\varepsilon a_{n+m}+b_{n+m}<-M a_{n}+b_{n} \text {. }
$$

Hence, in view of the monotonicity of $u_{n}(x)$ and (3.6) we get

$$
u_{n+m}\left(-\varepsilon a_{n+m}+b_{n+m}\right) \leq u_{n}\left(-M a_{n}+b_{n}\right) .
$$

By $n \rightarrow \infty, \varepsilon \rightarrow 0, M \rightarrow \infty$ we get $u(0-) \leq u(-\infty)=-\infty$, which contradicts condition (3.4).

Let $0<\beta<\infty$ and let us rewrite (3.6) as

$$
u_{n+m}\left(a_{n+m}\left(\frac{a_{n}}{a_{n+m}} x+\frac{b_{n}-b_{n+m}}{a_{n+m}}\right)+b_{n+m}\right) \leq u_{n}\left(a_{n} x+b_{n}\right) .
$$

Assuming $\alpha=\infty$ we get

$$
\left(b_{n}-b_{n+m}\right) / a_{n+m} \rightarrow \infty
$$

and, consequently, for every $\varepsilon>0$

$$
\frac{a_{n} \varepsilon}{a_{n+m}}+\frac{b_{n}-b_{n+m}}{a_{n+m}} \rightarrow \infty
$$


By (3.7) we come to a contradiction, since $u(+\infty) \leq u(\varepsilon)$ is impossible if $0<\varepsilon<\bar{u}$. Similarly, assuming $\alpha=0$ we will get for every $M>0$ the contradictory inequality $u(0) \leq u(-M)$. Finally, for $0<\beta<\infty, 0<\alpha<\infty$ we get from (3.7) the inequality $u(\alpha x+\alpha \beta) \leq u(x)$, which is also impossible if $u(x)$ satisfies condition (3.4). Thus we proved (3.5) and we conclude immediately that

$$
\lim \inf b_{n}>-\infty \text {. }
$$

Now, let us define the sequence $\left\{\beta_{n}\right\}$ as

$$
\beta_{n}=\inf \left(b_{n}, b_{n+1}, \ldots\right), \quad \dot{n}=1,2, \ldots
$$

Due to (3.8) $\beta_{n}>-\infty$. Obviously

$$
\beta_{n} \leq \beta_{n+1}, \quad \beta_{n} \leq b_{n}, \quad n=1,2, \ldots
$$

By Theorem 2.2, it is enough to prove that

$$
\left(b_{n}-\beta_{n}\right) / a_{n} \rightarrow 0 .
$$

Indeed, let us assume that for some $\beta>0$ and subsequence $\left\{n^{\prime}\right\}$ we have $\left(b_{n^{\prime}}-\beta_{n^{\prime}}\right) / a_{n^{\prime}}>\beta$. Then for every $n^{\prime}$ there exists among the terms of the reduced sequence $\left\{b_{n^{\prime}+m}\right\}(m=1,2, \ldots)$ a term $b_{n^{\prime}+m^{\prime}}$ such that

$$
\left(b_{n^{\prime}}-b_{n^{\prime}+m^{\prime}}\right) / a_{n^{\prime}}>\beta>0,
$$

which contradicts (3.5).

COROllary 3.1. Under the conditions of Lemma 3.3, there exists a $d f F^{*}(x)$ and a numerical sequence $\left\{b_{n}^{*}\right\}$ such that

$$
\begin{gathered}
u_{n}^{*}\left(a_{n} x+b_{n}^{*}\right)=n \frac{F^{*}\left(a_{n} x+b_{n}^{*}\right)-1}{\sqrt{ } g(n)}+\sqrt{ } g(n) \rightarrow u(x) \\
b_{n}^{*} \leq b_{n+1}^{*}, \quad n=1,2, \ldots
\end{gathered}
$$

and either

$$
b_{n}^{*} \rightarrow \infty, \quad \lim \inf b_{n}^{*} / a_{n} \geq-u
$$

or

$$
b_{n}^{*} \rightarrow 0, \quad a_{n} \rightarrow 0, \quad \lim \sup b_{n}^{*} / a_{n} \leq-\bar{u} .
$$

Proof. By Lemma 3.3, the initial $\left\{b_{n}\right\}$ may be replaced by a non-decreasing sequence $\left\{\beta_{n}\right\}$. Let $\beta_{n} \rightarrow b$, then $b>-\infty$.

If $b=\infty$, we put

$$
b_{n}^{*}=\beta_{n}, \quad F^{*}(x)=F(x) .
$$

Then inequality (3.10) follows from condition (3.4) and Lemma (3.1,1). 
If $|b|<\infty$, then we put

$$
b_{n}^{*}=\beta_{n}-b, \quad F^{*}(x)=F(x+b)
$$

and by Lemma $(3.1,2)$ we get $(3.11)$.

LEMMA 3.4. Under conditions (2.3)-(2.6), we have for every $v>1$

$$
\sqrt{ } g(n)\left(1-\frac{g(v n)}{v g(n)}\right) \rightarrow \infty
$$

Proof. For given $v>1$ and $M>0$ let $d>M v / \ln v$. Then from some $n$ on inequality (2.5) holds. Let us denote

$$
g(n ; v)=\max \{g(k), n \leq k \leq v n\} .
$$

(For simplicity we sometimes write $v n$ instead of $[v n]$ ). By (2.5), for sufficiently large $n$,

$$
g(v n) / g(n) \leq \prod_{k=n}^{v n-1}\left[1+\frac{1}{k}\left(1-\frac{d}{\sqrt{g(k)}}\right)\right]
$$

and therefore

(3.13) $\ln g(v n)-\ln g(n)<\sum_{k=n}^{v n-1} \frac{1}{k}\left(1-\frac{d}{\sqrt{ } g(k)}\right) \leq\left(1-\frac{d}{\sqrt{ } g(n ; v)}\right) \sum_{k=n}^{v n-1} \frac{1}{k}$

But

$$
\sum_{k=n}^{v n-1} \frac{1}{k}=\ln v+\frac{1}{2 n}(1-1 / v)+o(1 / n)<(1+1 / 2 n) \ln v+o(1 / n),
$$

since $\ln v>1-1 / v$ for $v>1$. Therefore, from some $n$ on,

$$
\sum_{k=n}^{v n-1} \frac{1}{k}<\left(1+\frac{1}{n}\right) \ln v<2 \ln v
$$

Obviously, again by (2.5), for $n$ large enough

$$
\ln g(n ; v)-\ln g(n)<\sum_{k=n}^{v n-1} \frac{1}{k}<\ln v^{2}
$$

and consequently

$$
1-\frac{d}{\sqrt{ } g(n ; v)}<1-\frac{d}{v \sqrt{ } g(n)}
$$

Thus by (3.13), from some $n$ on,

$$
\ln g(v n)-\ln g(n)<\left(1-\frac{1}{v \sqrt{ } g(n)}\right)\left(1+\frac{1}{n}\right) \ln v<\left(1+\frac{1}{n}-\frac{d}{v \sqrt{ } g(n)}\right) \ln v,
$$


i.e.

$$
1-\frac{g(v n)}{v g(n)}>1-\exp \left[\left(\frac{1}{n}-\frac{d}{v \sqrt{ } g(n)}\right) \ln v\right] .
$$

By (2.3) we have

$$
\sqrt{ } g(n) \ln v\left(\frac{d}{v \sqrt{ } g(n)}-\frac{1}{n}\right) \rightarrow \frac{d \ln v}{v}>M
$$

and therefore

$$
\lim \inf \sqrt{ } g(n)\left(1-\frac{g(v n)}{v g(n)}\right)>M
$$

where $M$ was taken arbitrarily. Thus we proved (3.12).

Lemma 3.5. Assume the conditions of Lemma 3.3 and in addition, let $\left\{b_{n}\right\}$ be non-decreasing. If for some $\beta(0<\beta<\bar{u})$ and for some integer valued function $m=m(n)>0$

$$
\left(b_{n+m}-b_{n}\right) / a_{n} \rightarrow \beta,
$$

then

$$
m(n) / n \rightarrow 0 .
$$

Proof. First we will prove that for every $v>1$

$$
\lim \inf \left(b_{n v}-b_{n}\right) / a_{n} \geq \bar{u} \text {. }
$$

Indeed, let us assume that for some subsequence $\left\{n^{\prime}\right\}$ and some $v>1$

$$
\left(b_{v n^{\prime}}-b_{n^{\prime}}\right) / a_{n^{\prime}} \rightarrow \ell<\bar{u} .
$$

By (3.13) we may assume that

$$
g\left(v n^{\prime}\right) / g\left(n^{\prime}\right) \rightarrow t^{2},
$$

where $0 \leq t^{2} \leq v$. Considering the sequences $\left\{u_{n^{\prime}}\left(a_{n^{\prime}} x+b_{n^{\prime}}\right)\right\}$ and $\left\{u_{v n^{\prime}}\left(a_{v n^{\prime}} x+\right.\right.$ $\left.\left.b_{v n^{\prime}}\right)\right\}$ and applying Theorem 2.2 we get

$$
\begin{aligned}
& L_{1}\left(n^{\prime}\right)=n^{\prime} \frac{F\left(b_{v n^{\prime}}\right)-1}{\sqrt{ } g\left(n^{\prime}\right)}+\sqrt{ } g\left(n^{\prime}\right) \rightarrow L_{1}, \\
& L_{2}\left(n^{\prime}\right)=n^{\prime} \frac{F\left(b_{v n^{\prime}}\right)-1}{\sqrt{ } g\left(v n^{\prime}\right)}+\frac{\sqrt{ } g\left(v n^{\prime}\right)}{v} \rightarrow L_{2},
\end{aligned}
$$

where $u(\ell-0) \leq L_{1} \leq u(\ell+0), L_{2}=u(0) / v$. But

$$
L_{1}\left(n^{\prime}\right)-\sqrt{g\left(v n^{\prime}\right) / g\left(n^{\prime}\right)} L_{2}\left(n^{\prime}\right)=\sqrt{ } g\left(n^{\prime}\right)\left(1-\frac{g\left(v n^{\prime}\right)}{v g\left(n^{\prime}\right)}\right) .
$$


Hence, by (3.18) and Lemma 3.4 we get that $L_{1}-t L_{2}=+\infty$, which is impossible, since $L_{1}$ and $L_{2}$ are finite numbers. Thus we proved (3.16).

Now, if for some subsequences $\left\{n^{\prime}\right\}$ we have $m\left(n^{\prime}\right) / n^{\prime}>a>0$, then $n^{\prime}+m\left(n^{\prime}\right)>v n^{\prime}$, where $v=a+1>1$. Hence we get

$$
b_{n^{\prime}+m\left(n^{\prime}\right)}-b_{n^{\prime}} \geq b_{v n^{\prime}}-b_{n^{\prime}},
$$

since $\left\{b_{n}\right\}$ does not decrease. In view of condition (3.14) we get a contradiction to $(3.16)$, since $0<\beta<\bar{u}$.

4. Proof of Theorem 1.4. It was noted in $\$ 2$ that it is enough to prove, that each proper $s f u(x)$, which is a limit of the sequence (2.9), must be of the form (2.11). Without loss of generality, we may assume that the limit $d f H(x)$, which appears in (2.6), is continuous at $x=0$ and $0<H(0)<1$. Hence, by $(2.7), u(x)$ satisfies conditions (3.4). Therefore, by Corollary 3.1, we may assume that the normalizing numerical sequences $\left\{a_{n}, b_{n}\right\}$ satisfy the particular conditions (3.9) and neither (3.10) or (3.11).

Let $\beta(0<\beta<\bar{u})$ be any number and let us consider the sets of integers $k$

$$
E(n ; \beta)=\left\{k:\left(b_{n+k-1}-b_{n}\right) / a_{n} \leq \beta\right\}, \quad n=1,2, \ldots,
$$

which are, obviously, not empty. There exists an $N=N(\beta)$ such that $E(n ; \beta)$ is bounded for $n \geq N$. Indeed, in case (3.10) we can take $N=1$, since $b_{n} \rightarrow \infty$, while in case (3.11) $b_{n} \rightarrow 0$ and, from some $n$ on, we have $-b_{n} / a_{n}>\beta$.

For a fixed $\beta$ let us define an integer valued function $m=m(n)$ by

$$
m=\max E(n ; \beta)
$$

for $n \geq N$ and, e.g., $m(n)=1$ for $n<N$. Then from some $n$ on we have

$$
\beta<\left(b_{n+m}-b_{n}\right) / a_{n} \leq \beta+\frac{b_{n+m}-b_{n+m-1}}{a_{n+m}} \frac{a_{n+m}}{a_{n}}
$$

i.e. also

$$
\beta a_{n} / a_{n+m}<\left(b_{n+m}-b_{n}\right) / a_{n+m} \leq \beta a_{n} / a_{n+m}+\left(b_{n+m}-b_{n+m-1}\right) / a_{n+m} .
$$

We will prove, that by our assumptions

$$
\lim \sup a_{n+m} / a_{n}<\infty .
$$

Indeed, if for some subsequence $\left\{n^{\prime}\right\}, m^{\prime}=m\left(n^{\prime}\right)$,

$$
a_{n^{\prime}+m^{\prime}} / a_{n^{\prime}} \rightarrow \infty
$$

then by (4.3), (2.12) and Theorem 2.2

$$
u_{n^{\prime}+m^{\prime}}\left(a_{n^{\prime}+m^{\prime}} x+b_{n^{\prime}}\right) \rightarrow u(x) .
$$

On the other hand, by (4.5), for any continuity points of $u(x) M<\varepsilon<0$ we 
have for sufficiently large $n^{\prime}$

Hence, by (3.6),

$$
a_{n^{\prime}+m^{\prime}} \varepsilon<M a_{n^{\prime}}
$$

$$
u_{n^{\prime}+m^{\prime}}\left(a_{n^{\prime}+m^{\prime}} \varepsilon+b_{n^{\prime}}\right) \leq u_{n^{\prime}}\left(a_{n^{\prime}} M+b_{n^{\prime}}\right) .
$$

Therefore, in view of (4.6) we get $u(\varepsilon) \leq u(M)$ i.e. $u\left(0_{-}\right)=-\infty$, which contradicts (3.4).

Now, using Lemma 3.5 and (2.12) we conclude from inequalities (4.2) and (4.4), that the function $m=m(n, \beta)$, which was defined by (4.1), satisfies conditions (3.14)-(3.15).

In view of $(3.15)$ we have

$$
\lim \sup g(n+m) / g(n) \leq 1,
$$

since by (2.5) $g(n+m) / g(n)<(n+m) / n$. On the other hand

$$
\lim \sup g(n+m) / g(n) \geq 1 \text {, }
$$

since $g(n) \rightarrow \infty$ and $m(n) \geq 1$. Thus there exists a subsequence $\left\{n^{\prime}\right\}$ such that

$$
g\left(n^{\prime}+m^{\prime}\right) / g\left(n^{\prime}\right) \rightarrow 1 .
$$

Considering the expression of $u_{n+m}(x)$ and taking into account relations (3.15) and (4.7) we get

$$
u_{n^{\prime}}\left(a_{n^{\prime}+m^{\prime}} x+b_{n^{\prime}+m^{\prime}}\right)-L\left(n^{\prime}\right) \rightarrow u(x),
$$

where

$$
L(n)=\frac{n}{\sqrt{ } g(n)}[g(n) / n-g(n+m) /(n+m)] .
$$

But by (3.14) we have also

$$
u_{n}\left(a_{n} x+b_{n+m}\right) \rightarrow u(x+\beta) .
$$

Therefore, if $x=\beta$ is a continuity point of $u(x)$, then from (4.8)-(4.9) we get

$$
L\left(n^{\prime}\right) \rightarrow u(\beta)-u(0)
$$

and consequently

$$
u_{n^{\prime}}\left(a_{n^{\prime}+m^{\prime}} x+b_{n^{\prime}+m^{\prime}}\right) \rightarrow u(x)+u(\beta)-u(0) .
$$

The limit $v(x)=u(x)+u(\beta)-u(0)$ is a proper $s f$. Therefore, by (2.9) and Theorem 2.1, $v(x)$ must be of the same type as $u(x)$. Thus we proved, that for every continuity point $\beta$ of $u(x), 0<\beta<\bar{u}$, there exists an $\alpha=\alpha(\beta)>0$ such that

$$
u(\alpha x+\beta)=u(x)+u(\beta)-u(0)
$$

at each continuity point $x$ of $u(x)$. 
Assuming $w(x)=u(x)-u(o)$, we get the equation

$$
w(\alpha x+\beta)=w(x)+w(\beta) .
$$

It is known $([1], \S 2)$, that each proper solution of the above equation must be of the form (2.11). On the other hand, if $w(x)$ is an $s f$ of the form (2.11), then for every constant $c$ the $s f u(x)=w(x)+c$ is of the same type as $u(x)$.

This completes the proof of our theorem.

ACKNOWLEDGEMENTS. This work was carried out while the author was on Sabbatical leave at the University of Toronto, Ontario, Canada.

I would also like to thank Professor H. Kesten of Cornell University for reading the manuscript and for his comments.

\section{REFERENCES}

1. Cheng, B. (1964). The limiting distributions of order statistics. Acta Math. Sinica 14, 694-714. Translation in Chinese Math. (1965) 6, 84-104.

2. Chibisov, D. M. (1964). On limit distributions for order statistics. Theory Prob. and its Applications 9, 142-148. (English translation).

3. Gnedenko, B. (1943). Sur la distribution limit du maximum d'une série aléatoire, Ann. Math. 44, 423-453.

4. Gnedenko, B. V. and Kolmogorov, A. N. (1954). Limit distributions for sums of independent random variables. Addison-Wesley, Cambridge Mass.

5. Mejzler, D. (1956). On the problem of the limit distributions for the maximal term of a variational series. Lvov. Politehn. Inst. Naučn. Zap. Ser. Fiz.-Mat. 38, 90-109 (Russian).

6. Smirnov, N. V. (1949). Limit distributions for the terms of a variational series. Trudy Mat. Inst. Steklova 25 (Russian). Amer. Math. Soc. translations 11, (1962), 82-143.

7. Smirnov, N. V. (1966). Convergence of distributions of order statistics to the normal distribution. Izv. Akad. Nauk Uzbek. SSR Ser. Fiz.-Mat. Nauk 10 (3), 24-32 (Russian).

8. Smirnov, N. V. (1967). Some remarks on limit laws for order-statistics. Theory Prob. and its Applications 12, 337-339. (English translation).

\footnotetext{
INSTITUTE OF MATHEMATICS

THE HEBREW UNIVERSITY

JERUSALEM, ISREAL
} 